\title{
Automated Feature Description of Follicle Size in Assisted Reproductive Treatment
}

\section{Musab Sahrim ${ }^{1}$, Ummu Solehah A.Rahman ${ }^{1}$, Wan Zakiah Wan Ismail ${ }^{1}$, Irneza Ismail $^{1 *}$, Juliza Jamaludin ${ }^{1}$, Sharma Rao Balakrishnan ${ }^{1}$}

${ }^{1}$ Program of Electronics Engineering, Faculty of Engineering and Build Environment (FKAB),

Islamic Science University of Malaysia (USIM), 71800 Bandar Baru Nilai, Negeri Sembilan, MALAYSIA.

Received 4 February 2018; accepted 10 April 2018, available online 30 April 2018

\begin{abstract}
In assisted reproductive treatment, monitoring of follicular size using serial ultrasound is essential to access ovarian response. Traditional method requires doctors to measure the follicle size manually which will lead to inaccurate findings. As for more consistent and reliable parameter of follicular growth, an automated feature description may offer better accuracy in estimating to the response. In this study, by using two-dimensional ultrasound to acquire data from the ovaries, the ultrasound result will indicate the feature description automatically without manual calculation. This automated feature description is developed based on image processing technique using canny edge-detection method in MATLAB. It provides the analysis of the features based on area, perimeter, compactness, major and minor axis and centroid dataset to identify the follicle size
\end{abstract}

Keywords: Renewable fuel, ethanol, numerical simulation, auto ignition, single-step mechanism

\section{Introduction}

Ultrasound method has been used in a variety of clinical settings to diagnose conditions of human organs and soft tissues such as heart, uterus and ovary [1]. In gynaecology, monitoring the conditions of follicles in women's ovary is very important in reproductive system study [2] by Maryruth Pradeepa Raghavan. Although the method using two-dimensional ultrasound is commonly used in reproductive treatment, there are several discrepancies when applying the ultrasound method including speckle, the condition of tissue texture, unclear edge and artefacts from the ultrasound image processing [3].

Although the method using two-dimensional ultrasound is commonly used in reproductive treatment, unfortunately little awareness has been given towards the improvement of this method. Several methods are used to detect the follicle such as using optimal thresholding method [4-5], active contour [6], region growing [7-8], cellular automata and cellular neural networks [9] and watershed [10]. Our approach to this project is inspired by the observations that the method used today imposes doctors to calculate the follicle size manually leading to time consuming. The objective of this study is to develop a two-dimensional segmentation algorithm for measuring follicular size based on automated feature description of follicles inside the ovary. Along this study, the suitable techniques for pre-processing stage, postprocessing stage and feature extraction stage are identified and categorized as research methodology.

In this study, image processing technique using MATLAB has been featured, so that the accuracy of follicular size measurement can be achieved. The data set of area and perimeter of follicle will be automatically displayed on the ultrasound image, in order to calculate the compactness of follicle and monitor its maturity. The segmentation method used is based on edge-based method. The process starts by manually selecting the region of interest (ROI) and deleting the unwanted part which called noise. The first step involved in this project is Image Acquisition. This step is used to read and display the image before being used for the next step which is Pre-Processing Step. PreProcessing Step involves segmentation and edgedetection which is used to select the region of interest; follicles.

\section{Image Segmentation Techniques}

Image segmentation techniques can be classified in three main stages; image acquisition, pre-processing stage and post-processing stage. The algorithm based on image segmentation techniques is used to develop a twodimensional segmentation for measuring follicular size based on automated feature description of follicles inside the ovary.

STEP 1: READ IMAGE. The first step is to read image from graphic files. In this project, the process starts by reading the follicles image in .jpg file format. The syntax that is used to read the follicles image is shown below. The function imread reads a grayscale or color image from the file specified by the filename. The full pathname needs to be specified if the file is not in the current folder, or in a folder on the MATLAB path.

I = imread ('folli12.jpg'); \%read image 
STEP 2: SHOW IMAGE. The image can be displayed by using imshow function with syntax shown below. It can be a grayscale, RGB (true color), or binary image. For binary images, imshow displays pixels with the value 0 (zero) as black and 1 as white, whereby grayscale and RGB display pixels with value from 0 to 255 . The title for the displayed image also can be shown to justify the image.

figure, imshow (I); title ('original')

STEP 3: ENHANCE IMAGE. In order to enhance the image, the function imadjust is used to control the value of image intensity from RGB to grayscale form. This function helps to increase the contrast of the output image. The coding generated to convert the image into grayscale, at the same time adjusting the enhancement of the follicles image is shown below.

\%adjusting the image enhancement

$[$ ht, wd, co $]=\operatorname{size}(\mathrm{I})$;

if co $<3$

$\mathrm{A}=$ imadjust (I, [0.5 0.8], []);

else

A = imadjust (rgb2gray (I), [0.1 0.11$],[])$;

$\%$ convert RGB to grayscale image

end

STEP 4: FILTER IMAGE. In order to remove noise in the input image, imfilter function is used. From the syntax shown, multidimensional B image is filtered with the filter $\mathrm{C}$ which is applying fspecial function and each elements is computed to become output $\mathrm{C}$. Other array function used in this project to filter the follicles image is ones function.

\%averaging filter

$\mathrm{c}=$ fspecial ('average', [ [1 1]);

$\mathrm{C}=$ imfilter $(\mathrm{B}, \mathrm{c})$;

STEP 5: CHANGE IMAGE TO BINARY IMAGE. This function im $2 \mathrm{bw}$ is used to convert grayscale image to binary image. The output image $\mathrm{D}$ replaces all pixels in the input image $\mathrm{C}$ with the value 1 (white) and 0 (black). $\mathrm{D}=\mathrm{im} 2 \mathrm{bw}(\mathrm{C})$;

STEP 6: EDGE DETECTION. This function takes a grayscale or a binary image $\mathrm{D}$ as its input, and returns a binary image $d$ of the same size as $D$, where the function finds edges in D. There are many edge-finding methods supported by this function such as canny, sobel and prewit, but in this project only introduces canny method as the edge obtained is suitable to the input image.

$\mathrm{d}=$ edge $(\mathrm{D}$, 'canny');

STEP 7: REMOVING SMALL OBJECTS. As the binary image $\mathrm{d}$ consist of some distortions, so to remove the noise, an image processing function bwareaopen is used which mean can take away all the objects containing fewer than limited pixels. In this project, less than 120 pixels image is automatically eliminated so that smooth follicles image can be obtained. The function used is shown below.

$\mathrm{bw}=$ bwareaopen $(\mathrm{d}, 120)$;

STEP 8: CROP IMAGE. To get the region of interest (ROI), the function imcrop is used to create an interactive Crop Image with the image displayed in the current figure, called the target image. The Crop Image tool is a moveable and the resizable rectangle that can be positioned.

crop $=$ imcrop $(\mathrm{bw})$

STEP 9: FILL IMAGE. Function imfill helps to fill holes in the binary image crop. A hole is a set of background pixels that cannot be reached by filling in the background from the edge of the image. This image then will be used in the next steps to be labelled or identified in order to extract several features of the follicles.

fill $=$ imfill (crop, 'holes');

STEP 10: LABELLING IMAGE. Function bwlabel returns a matrix $\mathrm{BW}$, of the same size as fill, containing labels for the connected objects in fill. Sometimes, to use less memory, bwlabel function is replaced with logical function in a call command.

$\mathrm{BW}=\operatorname{logical}($ fill);

vislabels(BW), title ('Each Object Labelled')

STEP 11: FIND BOUNDARIES. Function bwboundaries acts to trace the exterior boundaries of objects in the binary image BW. This function returns the label matrix $\mathrm{L}$ as the second output argument.

$[\mathrm{B}, \mathrm{L}]=$ bwboundaries (BW, 'noholes');

STEP 12: DETERMINE THE FEATURES OF OBJECT. The final algorithm is to measure the features of the processed image. The function measures a set of properties for each connected object in the binary image, BW.

$\mathrm{r}=$ regionprops(BW,'Area', 'Perimeter', 'Centroid', 'MajorAxisLength', 'MinorAxisLength'); 


\section{Results and Discussion}

In this project, the measurement of the follicles feature description is being done automatically using MATLAB. The result is being compared between two measurements; manual freehand technique and automated feature description using image segmentation technique. For manual freehand technique, the result is taken using Image $\mathbf{J}$ software where the region of interest in which measurement are selected and being drawn interactively for data processing. Meanwhile, for automated image segmentation technique, we use canny edge-detection to segment the follicles image and calculate the follicle feature description. Both results are determined from the number of pixels to measure the follicles area, perimeter, compactness and centroid.

\section{Manual Freehand Technique using ImageJ}

Current method used by medical practitioners involve manual freehand technique which produces less precise result. This technique is time consuming as the medical practitioners need to do extra works to find follicles measurements. Figure 1 shows the follicles image result from the manual freehand technique using Image J. The line is being drawn manually to get the edge of every follicles in ultrasound image, then the follicles are labelled accordingly before feature description being measured and recorded in table. Table 1 shows the result for manual follicular feature description measurement in pixel values.

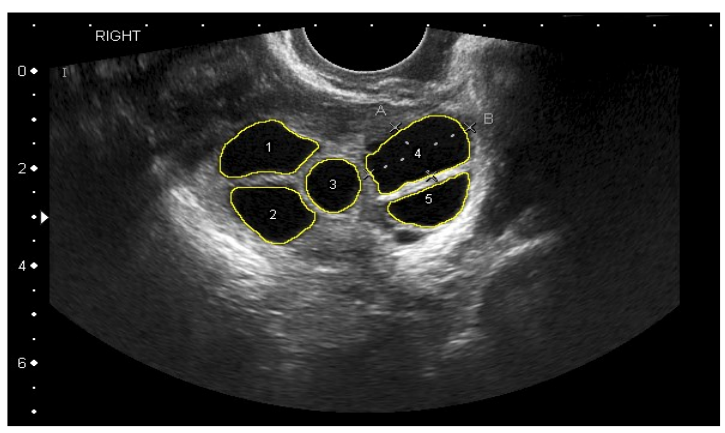

Fig. 1 Manual Freehand Technique using Image J.

Table 1 Manual Follicular Feature Description Measurement

\begin{tabular}{|c|c|c|c|c|}
\hline \multirow{2}{*}{$\begin{array}{c}\text { Labelled } \\
\text { Follicles }\end{array}$} & \multirow{2}{*}{$\begin{array}{c}\text { Area } \\
\text { (in }\end{array}$} & \multirow{2}{*}{$\begin{array}{c}\text { Perimeter } \\
\text { pixels) }\end{array}$} & & \multicolumn{2}{|c|}{ Centroid } \\
\cline { 4 - 5 } & in pixels) & $\mathrm{X}$ & $\mathrm{Y}$ \\
\hline 1 & 3052 & 224.66 & 275.72 & 202.72 \\
\hline 2 & 2706 & 203.32 & 281.94 & 262.10 \\
\hline 3 & 1812 & 156.63 & 332.58 & 235.82 \\
\hline 4 & 4045 & 258.94 & 403.88 & 204.74 \\
\hline 5 & 2062 & 193.36 & 415.42 & 253.54 \\
\hline
\end{tabular}

Automated Image Segmentation Technique using MATLAB

As the improvement from the traditional method using freehand, this project introduced an automated image segmentation technique using MATLAB in which the result is obtained after applying several steps involving image enhancement, image filtering, canny edgedetection segmentation, image labelling and feature extraction. Figure 2 shows the follicles image result from automated image segmentation technique using MATLAB.

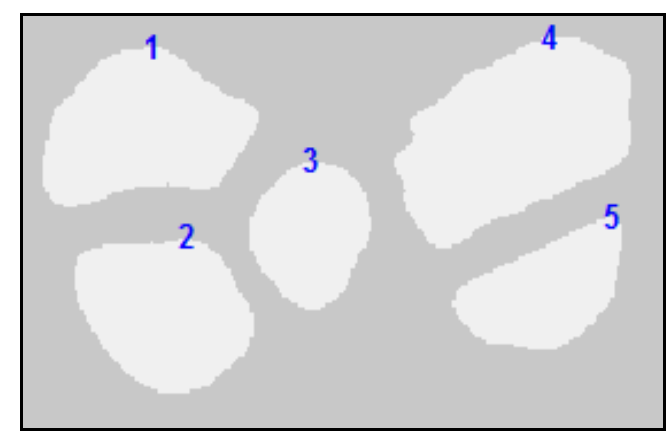

(a)

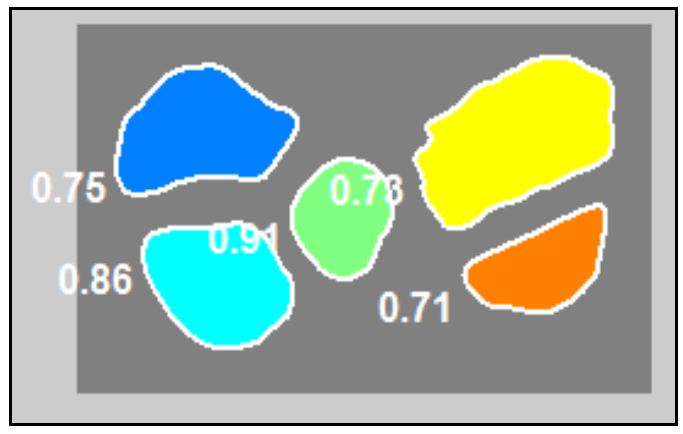

(b)

Figure 2: Automated Image Segmentation Technique using MATLAB. (a) Labelled follicles image, (b) Feature extraction of follicles image.

Figure 2(a) shows the labelled follicles in the original ultrasound image. These number of follicles then be measured to analyse the follicular feature description. Figure 2(b) shows the roundness of every follicles that is automatically calculated after getting the follicles boundaries. From the result obtained, metrics closer to one indicate that the object is approximately round. Thus, this shows that follicle number 3 is said to be the roundest shape compared to others. The result for every follicle feature description measurement in the form of pixel value is being recorded in Table 2 and Table 3 shows the result for follicles roundness measurement. 
Table 2 Automated Follicular Feature Description Measurement

\begin{tabular}{|c|c|c|c|c|c|c|}
\hline \multirow{2}{*}{$\begin{array}{c}\text { Label } \\
\text { Follicles }\end{array}$} & $\begin{array}{c}\text { Area } \\
\text { (in } \\
\text { pixels) }\end{array}$ & $\begin{array}{c}\text { Perimeter } \\
\text { (in } \\
\text { pixels) }\end{array}$ & $\begin{array}{c}\text { Major } \\
\text { Axis } \\
\text { Length }\end{array}$ & $\begin{array}{c}\text { Minor } \\
\text { Axis } \\
\text { Length }\end{array}$ & \multicolumn{2}{|c|}{ Centroid } \\
\hline 1 & 2662 & 210.65 & 75.08 & 47.47 & 48.17 & 41.34 \\
\hline 2 & 2312 & 183.92 & 62.98 & 47.42 & 55.86 & 100.29 \\
\hline 3 & 1477 & 143.20 & 47.32 & 40.07 & 106.53 & 74.58 \\
\hline 4 & 3634 & 250.11 & 91.85 & 51.31 & 177.27 & 43.59 \\
\hline 5 & 1568 & 166.61 & 63.61 & 33.14 & 187.94 & 93.36 \\
\hline
\end{tabular}

Table 3 Result for Follicles Roundness Measurement

\begin{tabular}{|c|c|}
\hline $\begin{array}{c}\text { Labelled } \\
\text { Follicles }\end{array}$ & Roundness \\
\hline 1 & 0.75 \\
\hline 2 & 0.86 \\
\hline 3 & 0.91 \\
\hline 4 & 0.73 \\
\hline 5 & 0.71 \\
\hline
\end{tabular}

\section{Summary}

Segmentation method used in this study is based on edgebased method where the objective is to develop an automated follicular feature description in assisted reproductive system. The features derived from the measurement of area, perimeter, compactness or solidity and centroid of the follicles. Analysis clearly indicates through accuracy that automated technique using MATLAB is the precise technique to detect follicles in ovary compared to manual freehand technique using Image J. This may help in identifying patients with risk of reproductive system in which early therapies may be beneficial.

\section{Acknowledgement}

This work was supported in part by project Supervisor, Dr. Musab Sahrim, beloved parents and family for excellent assistance and advice. A big thankful also is given to Dr. Mukhri Hamdan, Senior Lecturer and Fertility Specialist at University of Malaya Specialist Centre for supervising the work.

\section{References}

[1] Agarwal, A.K. Biofuels (alcohols and biodiesel) applications as fuels for internal combustion engines. Progress in Energy Combustion science, Volume 33, (2007), pp. 233-271.

[2] Dunphy, M.P., Patterson, P.M., and Simmie, J.M. High temperature oxidation of ethanol. Part 2Kinetic modeling. Journal of Chemical Society. Faraday Transactions, Volume 87, (1991), pp. 25492560.

[3] Egolfopoulos, F.N., Du, D.X., and Law, C.K. A study on ethanol oxidation kinetics in laminar premixed flames, flow reactors and shock tubes. Procceeding of Combustion Institute, Volume 24, (1992), pp. 833-841.

[4] Saxena, R., and Williams, F.A. Numerical and experimental studies of ethanol flames. Proceedings of Combustion Institute, Volume 31, (2007), pp. 1149-1156.

[5] Nortan, T.S., and Dryer, F.L. An Experimental and modeling study of ethanol oxidation kinetics in an atmospheric pressure flow reactor. International Journal of Chemical Kinetics, Volume 24, (1992), pp. 319-344.

[6] Marinov, N.M. A detailed chemical Kinetic model for high temperature ethanol oxidation. International Journal of Chemical Kinetics, Volume 31, (1999), pp. 183-220.

[7] Westbrook, C.H., and Dryer, F.L. Simplified reaction mechanisms for the oxidation of hydrocarbon fuels in flames. Combustion Science and Technology, Volume 27, (1981), pp. 31-43.

[8] Seiser R., Humer S., Seshadri K., and Pucher E. Experimental investigation of methanol and ethanol flames in non-uniform flows. Proceedings of Combustion Institute, Volume 31, (2007), pp. 11731180.

[9] Dubey, R., Bhadraiah, K., Raghavan, V., On the Estimation and Validation of Global Single-Step Kinetics Parameters of Ethanol-Air Oxidation Using Diffusion Flame Extinction Data. Combustion Science and Technology, Volume 183, (2011), pp. 43-50. 